\title{
A DEDICATED QUASAR MONITORING TELESCOPE
}

\author{
U. BORGEEST, K.-J. SCHRAMM AND J. VON LINDE \\ Megaphot, c/o Hamburger Sternwarte, Gojenbergsweg 112, \\ D-21029 Hamburg-Bergedorf, Germany; \\ uborgeest@hs.uni-hamburg.de, schramm@astra.astro.ulg.ac.be
}

\section{The Megaphot association}

Under the auspices of Sjur Refsdal, 25 astrophysicists and engineers from Germany and Scandinavia have founded a non-profit association, aiming at the use of an intelligent telescope for quasar monitoring in the optical (Borgeest et al. 1993). Beyond a better understanding of the physics in quasars, the scientific goals are determining the cosmic distance scale at large redshifts and constraining the nature of Dark Matter, both using the gravitational lens effect. Thus, targets of special interest are the multiply lensed quasars and some well-known violently variable blazars. The optical photometry will in part be carried out simultaneously to observations with, e.g., ISO, ROSAT, CGRO and various radio telescopes. For the first time, a complete quasar sample will be monitored continuously, namely a subsample of the all-sky $1 \mathrm{Jy}$ catalogue $(5 \mathrm{GHz})$. Since we will collect about $10^{6}$ photometric data points during the programme, Megaphot has been chosen as name for the association. Members from Hamburg and Bochum intend to test the $1.5 \mathrm{~m}$ Hexapod Telescope (HPT) astronomically in the very near future. The HPT hardware was developed and built by Vertex Antennentechnik, Duisburg together with the Ruhr-Universität, Bochum and Carl Zeiss, Jena; the intelligent software and weather control requires still some work. When working well, the system will be placed at a site with excellent astronomical conditions. After a few years of exclusive quasar monitoring, it will be used as a German photometry telescope.

\section{Hamburg Quasar Monitoring and Joint Monitoring}

Some Megaphot members are actually involved in quasar monitoring programmes using existing multi-purpose telescopes; e.g., the Hamburg Quasar 


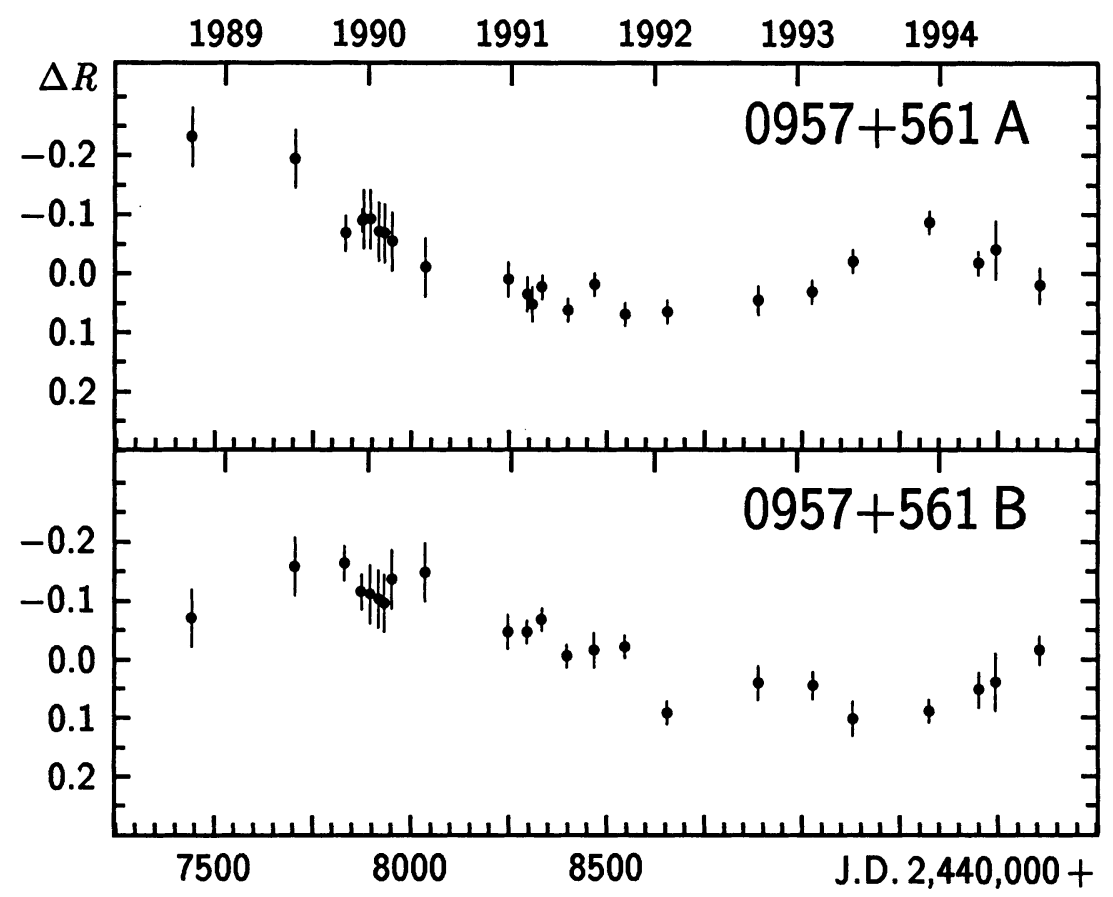

Figure 1. Results from automatic CCD photometry of the Double Quasar images, A and $B$, relative to stars in the field. Plotted is the mean for each observing campaign of typically 5 nights. Rough error estimates are indicated. Our CCD frames form a data set allowing a determination of the time delay $\Delta t$ between A and B for a time interval fully independent of the measurements of Vanderriest et al. (see Pelt et al., this Symposium). For the plotted lightcurves, a correlation by eye suggests $\Delta t \approx 425$ days rather than values above 500 days. However, a reliable error calculation for $\Delta t$ will only be possible after a more critical inspection of the single CCD frames.

Monitoring (HQM) programme at Calar Alto (since autumn 1988, Borgeest \& Schramm 1994) and an international Joint Monitoring Programme (since summer 1994, Schramm et al. 1994). For both, we use an automatic routine reducing the CCD frames immediately after the exposure. As an example, we show in Fig. 1 the HQM lightcurve of the gravitationally lensed Double Quasar $0957+561$. The time interval is independent from other, previously published data. A correlation by eye yields a time delay of $\approx 425$ days.

\section{References}

Borgeest, U., Schramm K.-J. (1994) The Hamburg Quasar Monitoring Program (HQM) at Calar Alto: I. Low amplitude variability in quasars, $A \& A 284,764$

Borgeest, U., Schramm K.-J., von Linde, J. (eds.) (1993) The Need for a Dedicated Quasar Monitoring Telescope; Proc. $1^{\text {st }}$ Megaphot Workshop. Megaphot, Hamburg

Schramm, K.-J., Bian, Y., Borgeest, U., Swings, J.P. (1994) The Joint Monitoring Programme of Quasars in the Optical, submitted to Chinese Astronomy and Astrophysics 